\title{
Editorial: Polyploid Population Genetics and Evolution-From Theory to Practice
}

\author{
Abdulqader Jighly ${ }^{1 *}$, Richard J. Abbott ${ }^{2}$ and Hans D. Daetwyler ${ }^{1,3}$ \\ ${ }^{1}$ Agriculture Victoria, AgriBio, Centre for AgriBiosciences, Bundoora, VIC, Australia, ${ }^{2}$ School of Biology, University of \\ St Andrews, St Andrews, United Kingdom, ${ }^{3}$ School of Applied Systems Biology, La Trobe University, Melbourne, VIC, \\ Australia
}

Keywords: allopolyploids, autopolyploids, polyploidization, mathematical biology, simulation

\section{Editorial on the Research Topic}

\section{Polyploid Population Genetics and Evolution-From Theory to Practice}

Despite polyploids being widespread and of great importance in eukaryotic diversification, our understanding of the dynamics of the evolution and inheritance of polyploids is less advanced than for diploids. The challenges in studying the population genetics and evolution of polyploids reside in the presence of more than two homoeologous "diverged but related" chromosome copies in allopolyploids or homologous "identical" chromosome copies in autopolyploids. Moreover, diploidization processes following polyploidy trigger other challenges in inferring paleo-polyploidization or ancient polyploidization events, which complicate the study of diverged homo(eo)logous genes and modeling of ecological factors affecting polyploids and their interactions with diploid ancestors. Statistical methods originally developed for the diploid mode of inheritance are generally biased when analyzing polyploids creating an urgent need to develop new methods for studying the evolutionary dynamics and modes of inheritance of polyploids (Dufresne et al., 2014; Meirmans et al., 2018). The aim of this Research Topic is to enhance our current understanding of the population genetics and evolution of polyploids and to highlight the practical applications that flow from such understanding. The collection of 12 papers covers four main areas of investigation: (1) the establishment of polyploids and long-term evolutionary consequences of polyploidy; (2) the evolution of gene expression, gene families, and chromosomes in polyploids; (3) the development of novel statistical polyploid-friendly population genetics models; and (4) the practical applications of different statistical models in polyploid trait evolution, quantitative genetics, and plant breeding.

With regard to the first of these topics, Baduel et al. provide a comprehensive review of factors affecting the successful establishment of newly formed polyploids in the wild and the shortand long-term costs and benefits that emanate from polyploidy. In this context, they discuss recent relevant ecological, physiological, cytological and genomic research, and underline the "wondrous cycles" of polyploidy (Wendel, 2015) in which polyploidization repeatedly happens after diploidization events over long evolutionary timescales. The advantages and disadvantages of polyploidy are further considered by Gaynor et al., but from the standpoint of a macro-scale study of the effects of polyploidization on the geographical community structure of two widely distributed flowering plant families, the Brassicaceae and Rosaceae, both of which have experienced multiple rounds of polyploidization events in the past. By combining cytogeographical information with phylogenetic analyses of plant communities in these two families across the USA, they show that communities may be shaped in diverse ways by polyploidy, but that impacts of genome duplication are not clear cut and are lineage specific. They highlight the need for much greater information on 
ploidal variation across species' ranges to provide a deeper understanding of the effects of genome duplication on plant community structure.

Following polyploidization, alterations to chromosome number and structure as well as gene function, expression, and copy number may occur and feature prominently in diploidization (Ohno, 1970; Tate et al., 2009; Conant et al., 2014; Jighly et al., 2019). With regard to changes in chromosome number, Jelenić et al. develop a mitotic mathematical model to predict the chromosome loss rate in polyploids before testing it in polyploid cells of the yeast, Saccharomyces cerevisiae. The model depends on spindle dynamics and the maximum duration of mitotic arrest. They show that a small change in spindle assembly time can cause a massive increase in the rate of chromosome loss in tetraploid cells. Focusing on gene expression and function, Takahagi et al. analyze 727 previously published RNA sequence datasets of hexaploid wheat collected from different developmental stages, tissues, and environmental conditions to examine differences in expression profiles. They observe genes that are present and expressed in triplets, doublets, or specifically in one subgenome, contributing to broad biological functions and annotations. With regard to gene family changes, Mable et al. report an analysis of European diploid and tetraploid Arabidopsis lyrata and Arabidopsis arenosa populations to infer the complex evolution of the "S-receptor kinases" (SRK) gene family. This gene family is involved in the female component of genetically controlled self-incompatibility and is subject to strong balancing selection (Castric and Vekemans, 2007). In turn, they examine how the diversity of $S R K$ alleles in tetraploids compares with that in diploid relatives, whether there is increased trans-specific polymorphism in tetraploids for these genes, if introgression occurs among species and ploidy levels, and whether copy number variation exists among paralogs.

Developing and extending widely used diploid theories and statistical models to fit polyploids is an important aim of the Research Topic. Meirmans and Liu extend the widely used analysis of molecular variance (AMOVA) to autopolyploids. This can be regarded as a significant step forward, given that since AMOVA was first developed by Excoffier et al. (1992), it has been widely employed in analyzing the population genetics of diploids. Similarly, site frequency spectrum (SFS) based methods such as the neutrality (Tajima, 1989) and (Fay and $\mathrm{Wu}, 2000)$ as well as heterozygosity of allelic variant tests such as Tajima's estimator of nucleotide diversity (Tajima, 1983) are widely used in diploid population genetics. Together with other SFS methods applied to high-throughput sequencing data, Ferretti et al. extend their application to autopolyploid populations and discuss their bias when applied to small populations. Detecting gene copy number variation is one of the most challenging tasks in the population genetic analysis of autopolyploids, leading (Knaus and Grünwald) to develop an $\mathrm{R}$ package "VCFR" to infer copy number variation in polyploids. The novelty of their method is that it does not require including the copy number of genomic regions (or alleles) a priori, but instead, VCFR infers them depending on the frequency of the most abundant alleles. Bourke et al. review the existing methods applied to experimental autopolyploid populations, such as breeding populations. They focus on methods of genotyping of polyploids, physical and genetic mapping procedures, simulating polyploid breeding populations, and quantitative genetic analyses including quantitative trait loci (QTL) mapping, genome wide association studies (GWAS) and genomic prediction in polyploids.

The final papers that comprise this Research Topic focus on the applications of polyploid population genetics in plant breeding. Ferrão et al. use a large breeding population of 1,575 autotetraploid blueberry individuals to dissect the genetic basis of eight fruit related traits and detect QTL associated with genotyping-by-sequencing based single nucleotide polymorphism (SNP) markers. They call their SNPs twice, with diploid and tetraploid genotype coding to compare the effect of diploid-like calling on GWAS results. Diploid coding resulted in shorter linkage disequilibrium blocks and a much smaller number of significantly associated QTL indicating the importance of using a tetraploid model. As an alternative to using tetraploid SNP coding, Manrique-Carpintero et al. developed a dihaploid potato population and conducted QTL mapping for vigor, height, and different tuber traits. Finally, in an examination of a population of synthetic allohexaploid wheat (Triticum turgidum - AABB $\times$ Aegilops tauschii - DD), Jighly et al. divided the additive variance for 12 biotic and abiotic stresses among the 21 chromosomes representing the $\mathrm{A}, \mathrm{B}$, and $\mathrm{D}$ subgenomes. They found that the wild $\mathrm{D}$ subgenome had the highest contribution to the additive variance in most traits, while the A subgenome had the lowest. They also reported a weak but significant positive correlation between the cumulative size of each of three homoeologous chromosomes and their cumulative additive variance.

The articles published on this Research Topic provide a body of knowledge in the field of polyploid population genetics and evolution. Though much progress has been made in this area, many challenges remain. Of particular importance will be the further development of robust statistical models for polyploids and the effective and efficient simulation of their population genetic and genomic complexities (Dufresne et al., 2014; Jighly et al., 2018). This will allow the testing of models and assumptions under complex evolutionary and demographic scenarios with validation using empirical data. Given the economic, ecological, and evolutionary importance of polyploidy, further concentrated research efforts are required to advance population genetic theories and applications that relate directly to polyploid species.

\section{AUTHOR CONTRIBUTIONS}

AJ provided the first draft. RA and HD edited the manuscript and provided additional text. All authors revised and approved the manuscript for submission. 


\section{REFERENCES}

Castric, V., and Vekemans, X. (2007). Evolution under strong balancing selection: how many codons determine specificity at the female self-incompatibility gene SRK in Brassicaceae? BMC Evol. Biol. 7:132. doi: 10.1186/1471-214 8-7-132.

Conant, G. C., Birchler, J. A., and Pires, J. C. (2014). Dosage, duplication, and diploidization: clarifying the interplay of multiple models for duplicate gene evolution over time. Curr. Opin Plant Biol. 19, 91-98. doi: 10.1016/j.pbi.2014.05.008

Dufresne, F., Stift, M., Vergilino, R., and Mable, B. K. (2014). Recent progress and challenges in population genetics of polyploid organisms: an overview of current state-of-the-art molecular and statistical tools. Mol. Ecol. 23, 40-69. doi: $10.1111 / \mathrm{mec} .12581$.

Excoffier, L., Smouse, P. E., and Quattro, J. M. (1992). Analysis of molecular variance inferred from metric distances among DNA haplotypes: application to human mitochondrial-DNA restriction data. Genetics 131, 479-491.

Fay, J. C., and Wu, C. I. (2000). Hitchhiking under positive darwinian selection. Genetics 15, 1405-1413. Available online at: http://www.genetics.org/content/ 155/3/1405.long

Jighly, A., Joukhadar, R., Sehgal, D., Singh, S., Ogbonnaya, F. C., and Daetwyler, H. D. (2019). Population-dependent reproducible deviation from natural bread wheat genome in synthetic hexaploid wheat. Plant J. 100, 801-812. doi: 10.1111/tpj.14480

Jighly, A., Lin, Z., Forster, J. W., Spangenberg, G. C., Hayes, B. J., and Daetwyler, H. D. (2018). Insights into population genetics and evolution of polyploids and their ancestors. Mol. Ecol. Res. 18, 1157-1172. doi: 10.1111/1755-0998. 12896

Meirmans, P. G., Liu, S., and van Tienderen, P. H. (2018). The analysis of polyploid genetic data. J. Hered. 109, 283-296. doi: 10.1093/jhered/esy006

Ohno, S. (1970). Evolution by Gene Duplication. New York, NY: Springer-Verlag.

Tajima, F. (1983). Evolutionary relationship of DNA sequences in finite populations. Genetics 105, 437-460.

Tajima, F. (1989). Statistical method for testing the neutral mutation hypothesis by DNA polymorphism. Genetics 123, 585-595.

Tate, J. A., Joshi, P., Soltis, K. A., Soltis, P. S., and Soltis, D. E. (2009). On the road to diploidization? Homoeolog loss in independently formed populations of the allopolyploid Tragopogon miscellus (Asteraceae). BMC Plant Biol. 9:80. doi: 10.1186/1471-2229-9-80

Wendel, J. F. (2015). The wondrous cycles of polyploidy in plants. Am. J. Bot. 102, 1753-1756. doi: 10.3732/ajb.1500320

Conflict of Interest: The authors declare that the research was conducted in the absence of any commercial or financial relationships that could be construed as a potential conflict of interest.

Copyright () 2019 Jighly, Abbott and Daetwyler. This is an open-access article distributed under the terms of the Creative Commons Attribution License (CC BY). The use, distribution or reproduction in other forums is permitted, provided the original author(s) and the copyright owner(s) are credited and that the original publication in this journal is cited, in accordance with accepted academic practice. No use, distribution or reproduction is permitted which does not comply with these terms. 